\title{
Simple visual, choice and discrimination reaction times of young adults and cor- relation with intelligence quotient and working memory.
}

Sooriyakanthan $M^{1}$, Aravinthan $V^{1}$, Kajananan $S^{1}$, Thuvaraga $P^{1}$, Nimalan $S^{1}$, Sivapalan $K^{1}$. ${ }^{1}$ Dept. of Physiology, Faculty of Medicine, University of Jaffina

\begin{abstract}
Reaction time (RT) is important in learning. Relationship of RT to Intelligent Quotient (IQ) and Working Memory (WM) has not been studied in Sri Lankans.
\end{abstract}

To measure the visual RT(VRT), discrimination RT (DRT) and choice RT (CRT) of young adults and to correlate these reaction times (RTs) to IQ and WM.

A cross sectional study was conducted among 200 volunteers (18-28 years). RTs were measured using the "reaction timer software" developed locally. WM was assessed using "digit span backward test (DSBT)". IQ was assessed using Raven's standard progressive matrices (RSPM).

Mean, SD of VRT, CRT and DRT in males were $292.85 \pm 32.88 \mathrm{~ms}, 395.75 \pm 75.62 \mathrm{~ms}$ and $407.45 \pm 82.11 \mathrm{~ms}$ respectively. The respective values in females were $317.85 \pm 43.15 \mathrm{~ms}$, $452.68 \pm 96.47 \mathrm{~ms}$ and $454.26 \pm 93.5 \mathrm{~ms}$. RTs were faster $(\mathrm{p}<0.05)$ in males. VRT was faster $(\mathrm{p}<0.05)$ than CRT and DRT in both sexes. CRT and DRT did not differ significantly in both sexes ( $p>0.05)$. Mean IQ scores of males and females were $52.17 \pm$ 6.2 and $51.74 \pm 6.2$. Respective DSBT scores were $7.55 \pm 1.62$ and $7.64 \pm 1.33$. IQ and DSBT scores were not differed $(p>0.05)$ between sexes. RTs had negative correlations with IQ and DSBT score. Only the correlations of IQ with VRT of males $(-0.203)$ and DRT $(-0.225)$ and CRT $(-0.235)$ of females were significant $(\mathrm{p}<0.05)$. Significant negative correlation $(-0.293)$ of DSBT score was observed with DRT of females only.
RTs were shorter in males. IQ and WM capacity showed negative correlations with RTs. Further studies are necessary to assess the contribution of the processing speed on the IQ and WMC.

\section{Key words}

Reaction times, Intelligent quotient, Working memory

\section{Introduction}

When a stimulus is applied, there will be a behavioral response. The time gap between the application of stimulus and appropriate voluntary response is called reaction time (RT). If the reaction is to simple visual stimuli, the time taken is called visual reaction time (VRT). Tasks are more complex in choice reaction time (CRT) and discriminative reaction time (DRT). RT involves the perception, information processing and coordinated peripheral movements. It is an important factor in day to day life for efficient interaction with environment and responding to it. RT is related to concentration, attention, arousal level and information processing which are important in carrying out learning (1).

Intelligence is ability to learn, understand or to deal with new situations which involves perception and information processing. Intelligence of a person is measured by a score called intelligent quotient (IQ). Whether people with faster reaction times have higher intelligence than those with slower RTs is unclear. It depends on whether the intelligence is decided by fast processing and transmission of impulses or correct processing and transmission which may be slower. Working Memory (WM) is 
an important factor to perform complex tasks like reasoning, comprehension and learning (2). RT comes faster with practice which could be due to learning and memory. Therefore, RTs, intelligence and WM are related to each other. According to the review published by Khodadadi et al, the correlation coefficient of many studies between RT and IQ had been significant but at different levels depending on study methodology, instruments used and data analysing (3).Correlation of RT with intelligence can be stronger in more complex RTs than simple RTs.

Only a few publications are available on SRT in Sri Lankans but correlation with IQ and WM is not available. Therefore, our study aims to measure the relationship between various types of visual RTs with IQ and WM. Young adults are selected as the study population because the above parameters reach optimal level in this population.

\section{Methodology}

This was a descriptive cross-sectional study conducted on healthy young adults aged $18-28$ years at Faculty of Medicine and Technical College in Jaffna. Getting admission to Medical faculty requires scoring top marks in highly competitive advanced level examination. Whereas, the qualification to get admitted to Technical College, can be less than ordinary level to pass in advanced level depending on the cause the student wants to follow. Therefore, participants were selected from these institutions expecting wide range of IQ. Subjects with a history of diabetes, ophthalmic diseases, neurological or any other defect in dominant upper limb and those on medication affecting cognitive performance were excluded from the study. Ethical clearance for the study was obtained from The Ethical Review Committee, Faculty of Medicine, Jaffna. Participation for the study was voluntary and informed written consent was obtained from every participant. Sample size was obtained by using the sample size formula for correlation studies: $\mathrm{N}=\left[\left(\mathrm{Z}_{\alpha}+\mathrm{Z}_{\boldsymbol{\beta}}\right) / \mathrm{C}\right]^{2}+$ 3. According to that $\mathrm{N}$ was 200 . Therefore, 100 males and 100 females were recruited.
Reaction times were measured using the "reaction timer software" developed locally and installed in a computer. Each subject was allowed to sit in front of the computer comfortably. Clear instruction and demonstration were given regarding the procedure.

For measurement of all the RTs, specific stimulus was generated by computer with random delay (maximum 10 seconds) after pressing the start button by the researcher. The visual stimulus was appearance of green color box in the monitor. For VRT, subject was asked to keep the dominant index finger on the space bar key and to press it as soon as possible when the stimulus arises. In CRT, the computer displayed an arrow which can be left pointing or right pointing and the participant was asked to press left or right arrow key with dominant index finger according to the display presented. In DRT, the participant was simultaneously presented with visual display of two circles with varied sizes and asked to press left or right arrow key with dominant index finger depending on left or right circle was larger. The timer displayed the time elapsed between the occurrence of stimulus and subject's response in milliseconds. Three readings were obtained in each type of RT and the minimum reading was considered for analysis.

IQ was measured by using Raven's Standard Progressive Matrices (RSPM). The RSPM kit was purchased from Pearson India education services Ltd. The test was done according to the instruction given. The test comprises five sets (A to E) of 12 diagrammatic puzzles in each (e.g., A1 through A12), with questions within a set becoming increasingly difficult. The questions consist of visual geometric design with a missing piece which the subject has to identify within the options provided. Subjects were given maximum one hour to complete all sets of questions. Score was calculated according to the responses. From the raw score, percentile rank was derived from the standard chart derived from US adult population because local standards are not available. According to the percentile rank each subject was categorized as Grade $1(\geq 95$ th percentile), Grade 2(75th to 94th percentile), Grade 
3 (25th to 74 th percentile), Grade 4 (5th to 24 th percentile) and Grade 5 ( $<5$ th percentile). Grades were considered as reflective of IQ of the subjects.

Digit span backward test (DSBT) was used to measure the WM. The test paper had two sets of digits for each span (ranging from 3 to 9 ). The procedure began by reading two sets of 3 digit span, one after the other, increasing the number of digits up to 9 . The participants were requested to write the readout digits in reverse order after 30 seconds of completing the reading. A time keeper was appointed to give the signal to commence paper-pencil contact. Participants were monitored by the investigators to make sure that the writing starts after the indication. Maximum of 30 participants were recruited at a time for the test. After completion of the task, the DSBT was scored manually. Of the two spans for each set, any one correct response was accepted. The participant was given the score of the highest correct response even if mistakes were made in sets of lower number of digits. The maximum band score in at least one of the two sets was accepted as reflective of the working memory capacity. Scores were categorized in to four bands based on the following: low ( $<3)$, average (4-5), above average (6-7) and high score ( $\geq 8)$.

Data was analyzed in SPSS 21. Student $t$ test was used to compare the significance of difference between males and females and to compare different reaction times. Spearman correlation was used to find out the significance of relationship between reaction times with IQ and WM.

\section{Results}

Number of participants in each age range is given in Table 1. The age range was based on WHO classification.

Table 1: Age distribution of the participants

\begin{tabular}{|l|l|l|}
\hline Age group (yrs) & Males & Females \\
\hline $18-22$ & 69 & 69 \\
\hline $23-27$ & 31 & 30 \\
\hline $28-32$ & & 01 \\
\hline
\end{tabular}

Vol.31, No.2, December 2019
Table 2 shows mean, SD of RTs of males and females and the $P$ value for sex difference.

Table 2: Mean \pm SD of reaction times of males and females

\begin{tabular}{|l|l|l|l|}
\hline RTs & Males & Females & Pvalue \\
\hline VRT & $292.85 \pm 32.88$ & $317.85 \pm 43.15$ & $<\mathbf{0 . 0 0 1}$ \\
\hline CRT & $395.75 \pm 75.62$ & $452.68 \pm 96.47$ & $<\mathbf{0 . 0 0 1}$ \\
\hline DRT & $407.45 \pm 82.11$ & $454.26 \pm 93.5$ & $<\mathbf{0 . 0 0 1}$ \\
\hline
\end{tabular}

All measured RTs were significantly faster in males than females $(p<0.05)$. VRT was faster than CRT and DRT which was statistically significant $(p<0.001)$ in both sexes. But, CRT and DRT did not differ statistically in both sexes $(p>0.05)$

Mean IQ scores of males and females were $52.17 \pm$ $6.2,51.74 \pm 6.2$. Mean grade levels of IQ were $2.7 \pm 0.98$ and $2.78 \pm 0.90$ in males and females respectively. This small difference in IQ between both sexes was not statistically significant $(p>0.05)$. Table 3 shows the distribution of IQ grades according to US standard.

Table 3: Distribution of IQ of males and females.

\begin{tabular}{|l|l|l|l|l|l|}
\hline Sex & \multicolumn{4}{|l|}{ Number of people } \\
\cline { 2 - 6 } & $\begin{array}{c}\text { Grade } \\
1\end{array}$ & $\begin{array}{c}\text { Grade } \\
2\end{array}$ & $\begin{array}{c}\text { Grade } \\
3\end{array}$ & $\begin{array}{c}\text { Grade } \\
4\end{array}$ & $\begin{array}{c}\text { Grade } \\
5\end{array}$ \\
\hline Males & 15 & 21 & 45 & 17 & 2 \\
\hline Females & 7 & 32 & 38 & 22 & 1 \\
\hline
\end{tabular}

The distribution shows that majority ( $45 \%$ of males and $38 \%$ of females) were in grade $3\left(25^{\text {th }}\right.$ to 74 percentiles) of IQ level. Only $2 \%$ of males and $1 \%$ of females of the study population fall in less than $5 \%$ (Grade 5$)$ of the IQ level.

DSBT score of males and females were $7.55 \pm 1.62$ and $7.64 \pm 1.33$ respectively and the difference was not statistically significant $(\mathrm{p}<0.05)$.DSBT score had correlations of $0.122(\mathrm{P}>0.05), 0.254(\mathrm{p}<0.05)$ with IQ score of males and females respectively. Distribution of DSBT score is shown in Table 4. 
Table 4: Distribution of DSBT of participants

\begin{tabular}{|c|c|c|c|c|}
\hline \multirow{2}{*}{ Sex } & \multicolumn{4}{|c|}{ Number of people } \\
\cline { 2 - 5 } & Low & Average & $\begin{array}{l}\text { Above } \\
\text { average }\end{array}$ & High \\
\hline Males & 0 & 19 & 24 & 57 \\
\hline Females & 0 & 9 & 36 & 55 \\
\hline
\end{tabular}

All the participants entered at least one span correctly. Majority (57\% of males and $55 \%$ of females) of the participants had high DSBT score. Table 5 shows the correlation co-efficient of RTs with IQ and DSBT score.

Table 5: Correlation coefficient between RTs, IQ and DSBT

\begin{tabular}{|c|c|c|c|c|}
\hline & \multicolumn{2}{|c|}{ IQ } & \multicolumn{2}{c|}{ DSBT } \\
\hline & Male & Female & Male & Female \\
\hline VRT & $-0.203^{*}$ & -0.183 & -0.090 & -0.173 \\
\hline DRT & -0.146 & $-0.225^{*}$ & -0.142 & $-0.293^{*}$ \\
\hline CRT & -0.168 & $-0.235^{*}$ & -0.107 & -0.030 \\
\hline
\end{tabular}

*- $p<0.05$

RTs had negative correlations with IQ and DSBT score. But only the correlations of IQ with VRT of males and DRT and CRT of females were statistically significant $(p<0.05)$. DSBT score had a significant negative correlation with DRT of females. No other significant correlations were observed between other RTs and DSBT score.

\section{Discussion}

Simple, choice and discriminative reaction times of young adults were measured in this study and they were correlated with IQ and WM. All RTs were faster in males than females, which is in conformity with earlier findings $(1,4)$. VRT was similar to earlier study in the same place but different subjects ${ }^{5}$ and higher than Indian studies $(4,5,6)$. CRT was higher than the values of Ritesh and Tegas in 2017 (7). Slower RT in this study may be due to differences in instruments or it could be real variation in different population. Faster VRT than CRT and DRT could be due to more processing time for complex tasks.
Distribution of IQ score in this population shows that only $2 \%$ of males and $1 \%$ of females are in grade 5 IQ score. This very lower $\%$ of population with lowest IQ grade could be due to the selective sampling of this study. As the participants are recruited from medical students and technical college students, more tendency is there for them to have relatively higher IQ than general population. As the main objective was to correlate IQ with RT, obtaining representative samples was not considered.

DSBT score shows no sex difference, suggesting working memory capacity of males and females are equal. This is in consistent with Nallaiah's finding in 2012 , Hill et alland Speck et al's in 2012, reported that different network systems in brain get activated during memory in both sexes $(8,9,10)$. This suggests that males and females show same memory capacity, achieved by activating different brain areas. This may also explain the equal IQ of males and females which could have been achieved by different problem solving strategies. As DSBT is an immediate verbal memory, there can be sex difference existing in other types of memory.

Although sex variation exists in RTs, no sex difference was observed in IQ and WM. This could be explained by that the RT varies due to movement time may not be due processing time. Hormonal influence after puberty and environmental opportunities supporting males cause better physical activities in males than females (11). It could have favored the RT in males.

Negative correlations with RTs and IQ show that people with higher IQ react faster. But these correlations were significant only with VRT in males and DRT and CRT of females. As DRT and CRT are more complex than VRT, it was expected that the IQ would correlate better with DRT and CRT than VRT. But this was observed only in females. This different pattern of observation in males and females is difficult to explain. Slower RTs in people with higher academic achievement was reported $(1,4)$. These studies were correlating the academic performance with RTs where as the present study correlates the IQ with the RTs. Although RT has 
negative correlation with IQ and as well as with academic performance, inferring the academic performance for IQ score is not accurate as academic performance can be influenced by various other factors. Correlations ofDSBT score with RTs were not statistically significant $(\mathrm{P}>0.05)$ except with DRT in females.

Our study had few limitations. RTs were measured using locally created software and no special devices were used in the measurements. Study group may have included athletes involving in different types of sport activities and non-athletes, which could have affected the reaction time.

\section{Conclusion}

RTs were shorter in males. IQ and WM capacity showed negative correlations with RTs even though all the correlations were not significant statistically. Further studies are necessary to assess the contribution of the processing speed on the IQ and WMC.

\section{Acknowledgements}

The authors acknowledge Mr. V. Sasikumar for developing the reaction timer software and Mr.S.B. Rameshkumar and the staff at the department of Physiology for their help in arrangements.

\section{References}

1. Taskin C. The visual and auditory reaction times of adolescents with respect to their academic achievements. Jeductrain stud 2016; 4(3):202-7.

2. Baddeley A. Working memory. Curr Biol 2010; 20 (4): R136- R140.

3. Khodadadi, M., Ahmadi, K., Sahraei, H., Azadmarzabadi, E., Yadollahi, S. Relationship between Intelligence and Reaction Time; A Review Study, International Journal of Medical Reviews 2014; 1(2). https://www.civilica.com/Paper-JR_IJMR-
JR_IJMR-1-2_001.html (accessed on 23 rd June 2018).

4. Prabhavathi,K., Hemamalini, RV., Thilip Kumar G, Amalraj, C., Maruthy KN., Saravanan A. A correlation study of visual and auditory reaction time with their academic performance among the first year medical students. Natl J Physiol pharm Pharmacol 2017; 7 (4): 371-4.

5. Balasubramaniam M, Sivapalan K, Nishanthi V, Kinthusa S, Dilani M.Effect of Dual-tasking on Visual and Auditory Simple Reaction Time. Indian J Physiol Pharmacol 2015; 59(2):194-8.

6. Karia RM., Ghuntla TP., Mehta HB., Gokhale, PA., Shah, CJ. Effect of Gender Difference on Visual Reaction Time: A Study on Medical Students of Bhavnagar Region. IOSR J Pharm 2012; 02: 452-4.

7. Ritesh KM.,\&Tegas., GP. Comparative study on simple and choice reaction time on medical students of Bhavanagar region. IRJP 2012;3: 334-5.

8. Nallaiah S. Influence of working memory capacity on academic achievement of final year medical students. IeJSME 2012; 06 (suppl 1): S 87-S102.

9. Hill, AC., laird AR., Robinson JL. Gender differences in working memory network: a brain map meta -analysis. Biol Psycol 2014; 102: 18-29.

10. Speck O, Ernst T, Braun J, Koch C, Miller E. Gender differences in the functional organization of the brain for working memory. Neuro report 2000; 11 (11): 2581-5.

11. Thomas, JR \&Thomas, KT. Development of Gender Differences in Physical Activity. Quest 1988; 40:219-29. 